\title{
The Application of Virtual Reality in Student Recruitment
}

\author{
Huiwen Zhao \\ Bournemouth University \\ Poole, UK \\ hzhao@bournemouth.ac.uk \\ Vedad Hulusic \\ Bournemouth University \\ Poole, UK \\ vhulusic@bournemouth.ac.uk
}

\author{
Alex Kelly \\ Bournemouth University \& Invidar \\ Poole, UK \\ alexkelly@invidar.com \\ Fred Charles \\ Bournemouth University \\ Poole, UK \\ fcharles@bournemouth.ac.uk
}

\begin{abstract}
In this paper we present details of a virtual tour and game for VR headset that are designed to investigate an interactive and engaging approach of applying VR to student recruitment for an undergraduate course. The VR tour employs a floating menu to navigate through a set of $360^{\circ}$ panoramic photographs of the teaching environment and uses hotspot interaction to display further information about the course. The VR game is a fast-paced shooting game. The course information is embedded on cubes that the player needs to focus on and destroy. The game experience is expected to generate an engaging way to promote the course. This work in progress outlines the concept and development of the prototype, and discusses the next stages of testing in order to evaluate the effectiveness of applying VR to undergraduate student recruitment.
\end{abstract}

\section{CCS CONCEPTS}

- Human-centered computing $\rightarrow$ Virtual reality.

\section{KEYWORDS}

Virtual Reality, VR Games, Student Recruitment

\section{ACM Reference Format:}

Huiwen Zhao, Alex Kelly, Vedad Hulusic, and Fred Charles. 2021. The Application of Virtual Reality in Student Recruitment. In 27th ACM Symposium on Virtual Reality Software and Technology (VRST '21), December 8-10, 2021, Osaka, Japan. ACM, New York, NY, USA, 2 pages. https://doi.org/10.1145/ 3489849.3489936

\section{INTRODUCTION}

Student recruitment is a key element in the sustainability and success of a higher education institution. With the development of immersive technology, VR has been considered as a powerful tool to connect with prospective students. In particular, VR tours provide the students with virtual experiences which allow them to explore the campus without actually being there.

A number of universities have already applied VR technology in their student recruitment campaigns [2]. A QS 2019 International

Permission to make digital or hard copies of part or all of this work for personal or classroom use is granted without fee provided that copies are not made or distributed for profit or commercial advantage and that copies bear this notice and the full citation on the first page. Copyrights for third-party components of this work must be honored

For all other uses, contact the owner/author(s).

VRST '21, December 8-10, 2021, Osaka, Japan

(C) 2021 Copyright held by the owner/author(s)

ACM ISBN 978-1-4503-9092-7/21/12.

https://doi.org/10.1145/3489849.3489936
Student Survey (ISS) showed that 39\% of students saw "virtual tours of the university" as being influential in their decision-making process [1]. Another study has also found that VR experience increases the average online engagement and inquiries [7]. It also leads to a $27 \%$ increase in physical visits to the universities after the VR experience.

Although VR technology helps with student recruitment, most of the existing work has been limited to creating VR tours. Given this consideration, in this paper we present a more interactive and engaging approach to promote the Games Design course at Bournemouth University in the UK, which does not only include the development of a $360^{\circ}$ VR tour but also a VR game. The $360^{\circ}$ VR tour aims to showcase basic information of the course as well as the teaching environment. The VR game allows students to further explore the course by presenting the units that are taught in an engaging way. The main challenge of the development is to balance the need to incorporate necessary course information for prospective students and to keep the experience interactive and engaging.

\section{DESIGN AND DEVELOPMENT}

\subsection{VR Tour}

We created a VR tour for 15 labs, lecture theatres and other teaching spaces. To help users transition between different rooms, a navigation menu is designed. Considering users are prospective students who may not have had a lot of VR experience before, the navigation menu is designed as a flat menu which is similar to the menu in $2 \mathrm{D}$ interfaces [3]. The menu was developed through an iterative design process. In the final design, it was decided to let the menu move with the user's head movement, i.e. the menu floats in 3D space and is activated via a ray-casting selection technique (Figure 1). To cater for variations in user height, a system was constructed that would set the height of the UI based on the position of the VR headset which, by design, would indicate the tallest point of the user, thus making the experience applicable for all regardless of height.

To provide additional information about each room and their use, hotspots are overlaid on the $360^{\circ}$ images. Hotspots can be selected via ray casting, which then presents the user with either a video or a text box with optional audio [4] (Figure 2). The system stores VR images and hotspot data separately, allowing the environment to be constructed upon startup. This gives us developmental flexibility and allows new scenes to be added quickly and efficiently. 


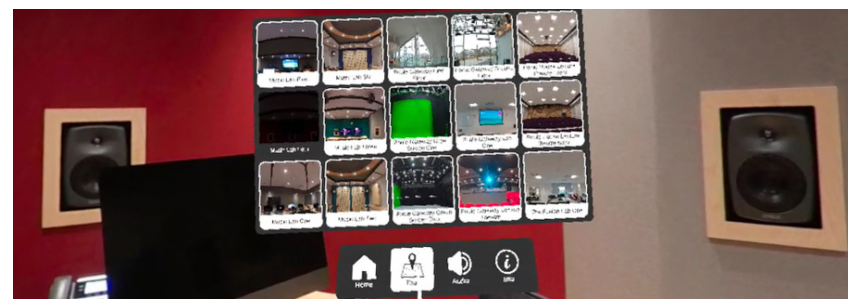

Figure 1: Navigation menu design.
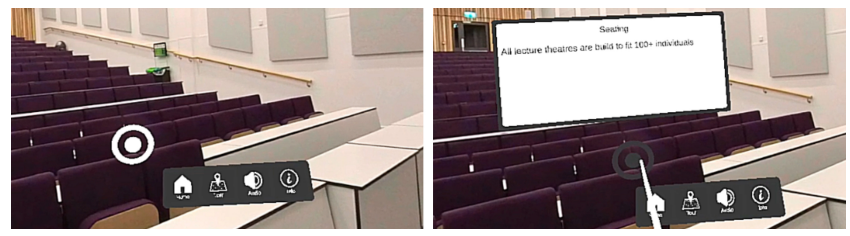

Figure 2: Hotspot interaction: before hotspot is active (left); with active hotspot (right).

\subsection{VR Game}

The main mechanics of the VR game are shooting and dodging. The reasons for creating an action-shooting game are because: first, according to Statista[6], action and shooters are two popular game genres in the UK. Second, action and shooters are fast paced which help attract players' attention in a short time, which is suitable for promotional purposes. In addition, shooting targets encourage players to focus on items used to convey course information to prospective students. Essentially, in the game space, there are small-sized target cubes and large-sized obstacle cuboids moving towards the player, which are also differentiated with different colour schemes. The surfaces of the target cubes show the names of the units on the course (Figure 3). Essentially, players need to pick up a virtual 3D gun to shoot target cubes while avoiding being hit by obstacle cuboids[5]. Players receive 10 points every time they manage to shoot a target cube and lose 10 points if they are hit by an obstacle cuboid. After a set number of points, the game will level up and the movement of cubes and cuboids becomes faster, with the 3D space also changing color to indicate the progression (Figure 4). The unit names on the target cubes then also progress to those from the next academic year.

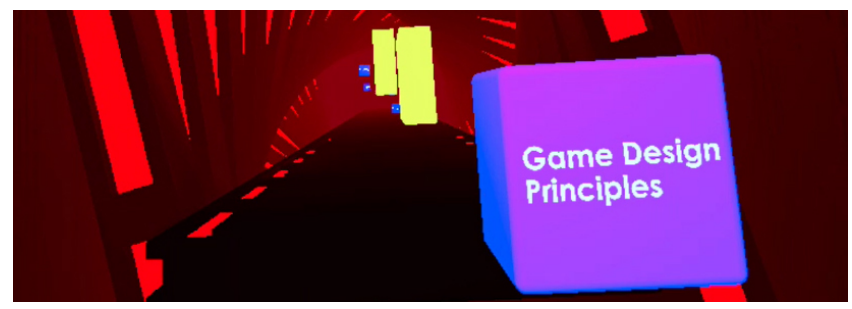

Figure 3: Purple target cubes and yellow obstacle cuboids are moving towards the player.

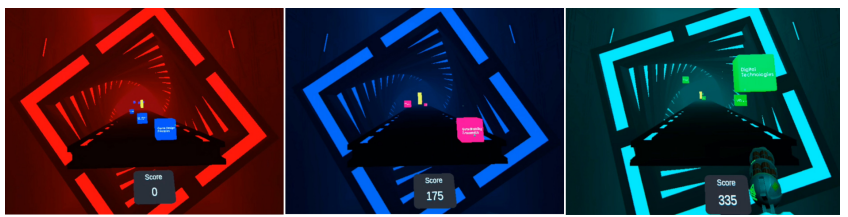

Figure 4: Interface design changes with level up.

Both the shooting and dodging mechanics rely on user position tracking. The system tracks only the headset and two hand controllers so full body tracking is unavailable for collision detection. Instead, user-obstacle collisions are detected with collision volumes attached to the hands and head. The hand collision volumes are coupled with Unity's XR management system to allow the user to pick up and use a virtual gun to fire at the target cubes.

The game's duration is based on the number of target cubes that are generated. Spawn points are in a grid layout facing the player across the game corridor, with the objects moving towards the player. Target cubes and obstacle cuboids are spawned from random spawn points at a ratio of 3:1. Spawn rate and movement speed are determined by the game level settings and are progressively increased with each level. Level progression and final win condition are based on points scored by the user for hitting the target cubes.

\section{CONCLUSIONS AND FUTURE WORK}

VR allows students to be transported to the heart of the university and taken on virtual journeys in an immersive way. This greatly benefits those who cannot travel to attend on-campus open days. Considering the impact of Covid-19, as well as other travel restrictions, VR has the potential to open up a new way for universities to communicate with prospective students. Both VR tours and VR games can be distributed online as a platform to communicate information and to interact between prospective students and staff. They can also serve as a demo booth for open days on campus, and VR headsets can be used to provide an immersive viewing experience. The next step for this project will be the evaluation of the effectiveness of applying VR to student recruitment.

\section{REFERENCES}

[1] 2019. How to Utilize Virtual Reality in Your Student Recruitment. Retrieved August 13, 2021 from https://www.qs.com/virtual-reality-student-recruitment/

[2] 2020. VR360 Video to Increase Student Recruitment and Enhance Student Experience. Retrieved August 13, 2021 from https://vipworldwide.tv/hotel-marketingblog/virtual-reality-360-video-tours-for-universities-to-increase-studentrecruitment/

[3] Doug A. Bowman and Chadwick A. Wingrave. 2001. Design and evaluation of menu systems for immersive virtual environment. In Proceedings IEEE Virtual Reality 2001. IEEE, 149-156.

[4] Julia Hayes and Kyungjin Yoo. 2018. Virtual reality interactivity in a museum environment. In Proceedings of the 24th ACM Symposium on Virtual Reality Software and Technology. ACM, New York, NY, USA, 1-2.

[5] Przemysław Krompiec and Kyoungju Park. 2019. Enhanced player interaction using motion controllers for first-person shooting games in virtual reality. In IEEE Access 7. IEEE, 124548-124557.

[6] Alexander Kunst. 2021. In general, which types of video games do you play? Retrieved October 6, 2021 from https://www.statista.com/forecasts/997858/videogame-preferences-by-genre-in-the-uk

[7] Sophie Thompson. 2020. VR in Higher Education: with Examples. Retrieved August 13, 2021 from https://virtualspeech.com/blog/vr-education-example-use-cases 